\title{
1 Prolactin mediates behavioural rejection responses to avian brood parasitism
}

3 Francisco Ruiz-Raya* ${ }^{* 1,2}$, Juan Diego Ibáñez-Álamo ${ }^{1}$, Charline Parenteau ${ }^{3}$, Olivier Chastel ${ }^{3}$

4 and Manuel Soler ${ }^{1}$

5

$6 \quad{ }^{1}$ Departamento de Zoología, Universidad de Granada, E-18071 Granada, Spain

72 Present address: Departamento de Ecología y Biología Animal, Universidad de Vigo, 836310 Vigo, Spain

$9{ }^{3}$ Centre d'Études Biologiques de Chizé (CEBC), UMR7372 CNRS - Université de La 10 Rochelle, France.

$13 *$ *Corresponding author: francisco.ruiz.raya@uvigo.es 


\section{Abstract}

26 Even though adaptations resulting from co-evolutionary interactions between avian

27 brood parasites and their hosts have been well studied, the hormonal mechanisms

28 underlying behavioural host defences remain largely unexplored. Prolactin, the main

29 hormone mediating avian parental behaviour, has been hypothesized to play a key role

30 in the orchestration of host responses to brood parasitic eggs. Based on the positive

31 association between plasma prolactin and parental attachment to eggs, decreasing levels

32 of this hormone are expected to facilitate egg-rejection decisions in parasitized clutches.

33 We tested this prediction by implanting Eurasian blackbirds (Turdus merula) females

34 with an inhibitor of prolactin secretion, bromocriptine mesylate, to experimentally low

35 their prolactin levels. We found that bromocriptine-treated females rejected mimetic

36 model eggs at higher rates than placebo-treated individuals. To our knowledge, this is

37 the first experimental evidence that host responses to brood parasitism are mediated by

38 the primary endocrine pathway that orchestrates the expression of avian parental care.

40 Keywords: Acceptance threshold, egg rejection, hormones, parental behaviour 


\section{Introduction}

48 Obligate avian brood parasites lay their eggs in the nest of host species, thus exploiting

49 the parental care that other birds provide to their offspring (Roldán and Soler, 2011).

50 This reproductive strategy imposes severe fitness costs on hosts since the newly-hatched

51 parasitic nestling often kills or outcompetes all host's offspring (Feeney et al., 2014;

52 Soler, 2014). In turn, hosts have evolved a wide range of behavioural, morphological

53 and life-story adaptations to counteract brood parasitism at all stages of the breeding

54 cycle (Feeney et al., 2014; Soler, 2014; Soler, 2017). Surprisingly, while behavioural,

55 cognitive and conditional aspects of host resistance are well known (Soler, 2017), we

56 still have a very limited understanding of the physiological mechanisms underlying anti-

57 parasitic host responses to avian brood parasitism.

58 Among anti-parasitic behaviours, the recognition and subsequent rejection of parasitic eggs is the most widespread defence used by hosts to counteract brood parasitism (Davies and Brooke, 1989; Feeney et al., 2014; Soler, 2014). At a mechanistic level,

61 egg rejection involves the selective disruption of typical parental behaviours to actively

62 respond against a specific stimulus from the own clutch (i.e. parasitic eggs), so it has

63 been hypothesized that host responses to parasitic eggs might be mediated by hormonal

64 pathways involved in the regulation of parental decisions (Abolins-Abols and Hauber,

65 2018; Ruiz-Raya and Soler, 2020). Recent evidence suggests that physiological and

66 behavioural host responses to foreign eggs may be mediated by the neuroendocrine

67 pathways underlying stress physiology (i.e. glucocorticoids (Abolins-Abols and Hauber,

68 2020; Ruiz-Raya et al., 2018)); however, whether rejection decisions are regulated by

69 the endocrine pathways mediating the expression of avian parental behaviours remains

70 unexplored. 
71 Prolactin, classically known as the "parental hormone" (Buntin, 1996; Sockman et al.,

72 2006), is the primary candidate for orchestrating anti-parasitic defences associated with

73 parental decisions (Abolins-Abols and Hauber, 2018; Ruiz-Raya et al., 2018). This

74 peptide hormone mediates the transition from sexual to parental activity in birds

75 (Buntin, 1996; Smiley, 2019; Sockman et al., 2006), playing a key role in the regulation

76 of major aspects of avian parental care: prolactin levels are positively related with the

77 initiation and maintenance of associative parental behaviours such as incubation or post-

78 hatching parental care (Angelier and Chastel, 2009; Angelier et al., 2016; Smiley,

79 2019). Importantly, the presence of foreign eggs has been found to lead to changes in

80 the prolactin response to stressors (Ruiz-Raya et al., 2018), which suggests that this

81 hormone might play a crucial role in mediating host responses to parasitic eggs

82 (Abolins-Abols and Hauber, 2018; Ruiz-Raya and Soler, 2020). Based on the positive

83 association between circulating prolactin and the expression of avian parental

84 behaviours (Angelier et al., 2016; Smiley, 2019), it may be hypothesized that a

85 reduction in prolactin levels would affect rejection decisions. Specifically, decreasing

86 prolactin levels would be expected to result in more restrictive egg acceptance

87 thresholds and facilitate rejection decisions (Abolins-Abols and Hauber, 2018). Here,

88 we tested this hypothesis for the first time by implanting host females with

89 bromocriptine-mesylate pellets, a dopamine receptor agonist, to experimentally decrease

90 their prolactin levels. Afterwards, we assessed their response to experimental parasitism

91 compared to placebo-implanted individuals in order to determine whether changes in

92 prolactin profiles affect hosts' rejection decisions.

\section{Material and methods}

\section{$94 \quad$ Study site and species}


95 This study was conducted in a Eurasian blackbird (Turdus merula) population located in

96 the Valley of Lecrín, Southern Spain, from late March to June 2018. The Eurasian

97 blackbird (hereafter blackbird) is a potential common cuckoo (Cuculus canorus) host

98 frequently used in egg-rejection studies (e.g. Grim et al., 2011; Roncalli et al., 2019;

99 Ruiz-Raya et al., 2015; Samas et al., 2011; Soler et al., 2015; Soler et al., 2017).

100 Blackbirds typically remove foreign eggs by grasping them with their bill (Soler et al.,

101 2015), showing high egg-ejection abilities (see references above).

102

103

104

105

106

107

108

109

110

111

112

113

114

115

116

117

118

119

\section{Hormone manipulation}

Females $(\mathrm{N}=36)$ were captured just after clutch completion (mean $\pm \mathrm{se}=2.3 \pm 0.19$ days) by using a mist net placed near the nest (6:00 - 8:00 am). Blood samples were collected from the brachial vein with a 25-gauge needle and $80-\mu l$ heparinized microhematocrit tubes within 3 minutes after capture. Through the breeding season, we sequentially assigned females to one of two experimental groups. Half of the females (n $=18)$ were implanted with bromocriptine mesylate $(\mathrm{BRC})$ time-release pellets (according to the characteristics of our study species: C-231, $0.5 \mathrm{mg}, 10$-days release, $3.1 \mathrm{~mm}$ diameter; Innovative Research of America), a dopamine receptor agonist which has been widely used to lower circulating prolactin in avian species (e.g. Angelier et al., 2006; Cottin et al., 2014; Smiley and Adkins-Regan, 2018; Thierry et al., 2013). Another half of the individuals $(\mathrm{n}=18)$ were assigned to the control group and therefore implanted with placebo pellets $(\mathrm{C}-111,0.5 \mathrm{mg}, 10$-day release, $3.1 \mathrm{~mm}$ diameter; same company). Time-release dosage pellets have been frequently used to modify hormone levels in ecophysiological studies, showing no negative long-term effect for birds' welfare (e.g. Cottin et al., 2014; Müller et al., 2009; Thierry et al., 2013). Even so, in order to discard the existence of unintended side effects of our BRC treatment on avian feeding behaviour (Buntin, 1989), females were weighted to the nearest $1 \mathrm{~g}$ before and 
120 after the hormonal treatment to assess potential changes in their body mass (Smiley and

121 Adkins-Regan, 2018). Additionally, although nest desertion is not an egg-rejection

122 mechanism in blackbirds (Soler et al., 2015), we evaluated whether our BRC

123 manipulation affected the probability of nest abandonment.

124 BRC and placebo-implanted individuals underwent identical procedures. Pellets were 125 implanted subcutaneously in the female's back through a small incision $(5 \mathrm{~mm})$ that was 126 subsequently sutured with surgical tissue adhesive (Vetbond, 3M). In our study 127 population, there was no seasonal variation in baseline prolactin in both experimental 128 groups (both cases $p>0.57$; Fig. S1; see statistical methods). Individuals were marked 129 with a combination of coloured rings to verify their identity. All birds were released 10 130 min after implantation. All females returned to their nest to resume incubation within 131 the next two hours. To test the effectiveness of the 10-day release BRC pellets, females $132(\mathrm{~N}=9)$ were recaptured six days after implantation (i.e. at the end of the experimental 133 parasitism trials; see below) and a second blood sample was collected from the opposite 134 wing. Similar sample sizes have been proved valid to verify hormone implants/injection 135 effectiveness in previous studies (Abolins-Abols and Hauber, 2020; Goutte et al., 2010; 136 Ouyang et al., 2013), so we minimized the number of re-sampled females to reduce the 137 stress linked to recapture and handling for breeding pairs. The visual examination of 138 recaptured individuals revealed that incisions were successfully sutured in all cases. 139 Blood samples were kept cold for up to 6 hours until centrifugation. Afterwards, plasma 140 was extracted and stored at $-20{ }^{\circ} \mathrm{C}$ until the hormonal assay. Prolactin plasma 141 concentration was determined by a heterologous radioimmunoassay at the Centre 142 d'Etudes Biologiques de Chizé. The assay was conducted using chicken prolactin and 143 antibody against chicken prolactin (supplied by Dr. A. F. Parlow, Harbor-UCLA 144 Medical Center, Torrance, CA, USA). All samples were run in one assay, in duplicate, 
145 with $25 \mu \mathrm{l}$ of plasma for each duplicate and a limit of detection of $0.43 \mathrm{ng} / \mathrm{ml}$. The intra-

146 assay coefficient of variation was $10.3 \%$.

\section{Brood parasitism experiments}

148 Nests were parasitized with mimetic eggs 24 hours after pellet implantation. As model 149 eggs, we used natural blackbird eggs collected from deserted clutches, which were 150 painted mimetic to simulate interspecific parasitism (Fig. 2b; see (Soler et al., 2015) for 151 additional details). This type of mimetic model eggs elicits intermediate ejection rates in 152 blackbirds (26.8 - 50\% (Roncalli et al., 2019; Soler et al., 2015)), thus allowing to detect 153 bi-directional changes in egg-rejection rates in response to the experimental 154 manipulation. All nests were checked every $24 \mathrm{~h}$ to determine egg ejection. Recent 155 research shows that nest desertion is not an egg-rejection mechanism in medium-sized 156 hosts such as blackbirds (Soler et al. 2015), so nest abandonment was not considered a 157 genuine response to experimental brood parasitism. Experimental eggs were consider as accepted if they remained in active nests for 5 days (Roncalli et al., 2019; Ruiz-Raya et al., 2015; Ruiz-Raya et al., 2016; Soler et al., 2017).

\section{Statistical analysis}

Between-groups differences in prolactin levels prior to our hormonal manipulation were assessed through a linear model including treatment, clutch size, and their interaction as predictors. We used linear regression to explore seasonal variations in baseline prolactin levels before implantation. Linear mixed-effect models were fitted by using nlme ( $\mathrm{R}$ package v.3.1-117 (Pinheiro et al., 2014)) to assess the effect of our manipulation on circulating prolactin and body mass. As fixed factors, our mixed models included: implant treatment (BRC or placebo), sample day, and their interaction, while female ID 
169 performed to assess the effect of our experimental treatment on egg ejection. As

170 predictors, our binomial regression model included: implant treatment, clutch size (3-4

171 eggs), the interaction between these two terms, baseline prolactin levels before

172 implantation and laying date. We assessed whether our BRC treatment affected nest

173 abandonment through a binomial regression model including implant treatment and

174 clutch size as predictors. Stepwise procedures were avoided to minimize the chances for

175 type I errors, and full models were used for inference (Whittingham et al., 2006).

176 Assumptions for normality of residuals and homogeneity of variances were verified by

177 the visual inspection of the residual graphs. All analysis and graphs were performed

178 using $\mathrm{R}$ version 3.6.1.

\section{Results and Discussion}

180 Pre-treatment prolactin levels did not differ between BRC and placebo-implanted

181 females (estimate $\pm \mathrm{se}=3.459 \pm 6.493 ; \mathrm{t}=0.533 ; p=0.60 ; \mathrm{N}=36$; Fig. 1a); however,

182 both groups differentially varied their prolactin levels in response to our BRC treatment

183 (implant treatment $\mathrm{x}$ sample day: $\mathrm{F}_{1,7}=52.17 ; p<0.001$; Fig. 1b). Specifically, while

184 BRC-treated individuals showed a significant reduction in their prolactin levels after

185 implantation (Tukey's test: estimate $\pm \mathrm{se}=35.39 \pm 4.02 ; \mathrm{df}=7 ; \mathrm{t}=8.81 ; p<0.001$ ),

186 circulating prolactin did not vary in placebo-implanted females (Tukey's test: estimate \pm

$187 \mathrm{se}=-8.12 \pm 4.49 ; \mathrm{df}=7 ; \mathrm{t}=-1.81 ; p=0.34)$. These results confirm the effectiveness of

188 our hormonal manipulation, and supports previous studies using BCR to experimentally

189 decrease plasma prolactin levels in birds (e.g. Angelier et al., 2006; Cottin et al., 2014;

190 Smiley and Adkins-Regan, 2018; Thierry et al., 2013).

191 Prolactin is the main hormone orchestrating the expression and maintenance of avian

192 parental behaviours during incubation (Angelier and Chastel, 2009; Smiley, 2019), so

193 egg-rejection decisions might be affected by circulating levels of this hormone 
194 (Abolins-Abols and Hauber, 2018; Ruiz-Raya and Soler, 2020). As expected, the 195 experimental decrease in circulating prolactin carried out in our study significantly 196 affected the host response to experimental brood parasitism: BRC-implanted females 197 ejected mimetic model eggs at significantly higher rates than placebo-implanted 198 individuals (implant treatment: $\chi^{2}=5.59$; $\mathrm{df}=1 ; p=0.018$; Fig. 2a), independently of 199 clutch size (implant treatment $x$ clutch size: $\chi^{2}=0.24 ; \mathrm{df}=1 ; p=0.62$ ). Laying date and 200 baseline prolactin levels did not affect the egg-ejection behaviour of females (both cases $201 p>0.76)$. These results show that the experimental reduction of plasma prolactin 202 increases the probabilities of egg rejection in blackbirds, while placebo-treated 203 individuals ejected mimetic model eggs at similar rates to those found in previous 204 studies in this species where no hormonal manipulation was performed (Soler et al., 205 2015). To the extent of our knowledge, this is the first experimental demonstration that 206 host responses to brood parasitism may be mediated by the main endocrine pathway 207 involved in the expression of avian parental care.

208 A better knowledge of the endocrine bases of host responses to brood parasitism will be 209 crucial to further understand individual variation in the expression and evolution of 210 rejection decisions (Abolins-Abols and Hauber, 2018). Since hosts might mistakenly 211 eject an own egg (recognition costs), or break their clutch during the process (ejection 212 costs) (Marchetti, 1992; Stokke et al., 2002), parasitized individuals are expected to 213 optimize their individual performance by adjusting their acceptance thresholds 214 according to host-parasite egg phenotype dissimilarity, previous experience or 215 parasitism risk (Hauber et al., 2006; Ruiz-Raya and Soler, 2017; Ruiz-Raya and Soler, 216 2020). While the endocrine mechanisms underlying conditional responses to avian 217 brood parasitism have been little studied, our results indicate that flexible shifts in 218 hosts' acceptance thresholds may be linked to variations in circulating prolactin. 
219 Interestingly, Abolins-Abols and Hauber (2020) have recently shown that an

220 experimental reduction in plasma corticosterone increases the acceptance of parasitic

221 model eggs in American robins (Turdus migratorius), a brown-headed cowbird

222 (Molothrus ater) host closely related to Eurasian blackbirds. Taken together, these

223 findings suggest that egg-rejection decisions are under the control of multiple endocrine

224 mechanisms, and draw attention to the need for integrative studies combining prolactin

225 and corticosterone, two hormones that may interact under certain circumstances to

226 maintain parent homeostasis, providing complementary information on parental

227 decisions (Angelier et al., 2016).

228 Beyond individual differences in baseline prolactin levels, interindividual variation in 229 egg-rejection decisions might be determined by differences in hormonal sensitivity to 230 environmental stimuli. Plasma prolactin has been found to decrease in response to 231 stressors in many bird species (Angelier and Chastel, 2009; Angelier et al., 2016), so the 232 host response to brood parasitism might be to some extend modulated by the strength of 233 the prolactin response to environmental stressors. For example, an increase in the 234 perceived risk of brood parasitism (e.g. witnessing a parasite near the nest) might lead 235 to a drop in host's prolactin levels, which would explain why the sight of a parasite at 236 the nest facilitates egg rejection by some hosts (Bartol et al., 2002; Moksnes et al., 237 1993; Moksnes et al., 2000). Under certain circumstances, hosts might be expected to 238 modulate their prolactin response to stressors according to current environmental 239 conditions, which could result in an adaptive prolactin stress response (Angelier and 240 Chastel, 2009). Some hosts rely on the combined use of personal and social information 241 about local parasitism risk to plastically adjust their acceptance thresholds to the current 242 environmental context (Thorogood and Davies, 2016), or they require from two stimuli 243 of brood parasitism (a parasitic egg in the nest and female parasite presence) to reach 
244 the threshold for egg rejection (Moksnes et al., 1993). Interestingly, the reduction in

245 plasma prolactin in response to stressors is more pronounced in individuals

246 experimentally parasitized with non-mimetic eggs (Ruiz-Raya et al., 2018), which could

247 suggest that prolactin responsiveness to parasite presence might be higher after

248 perceiving a first cue of brood parasitism (e.g. an odd egg in the nest). While brood

249 parasitism has been shown to impact the endocrine profiles of adult hosts during

250 incubation (Ruiz-Raya et al., 2018), nestling (Antonson et al., 2020) and fledgling

251 stages (Mark and Rubenstein, 2013), the extent to which adult parasite presence affects

252 the hormonal levels of hosts still needs further study. Furthermore, since circulating

253 prolactin often vary according to different life-history contexts (e.g. in response to

254 predation risk or unpredictable environmental conditions (Angelier and Chastel, 2009;

255 Angelier et al., 2016)), our understanding of the physiological mechanisms underlying

256 anti-parasitic defences would benefit from the study of the endocrine response of hosts

257 to their entire environmental context.

258 In our study, five out of 36 females (13.8\%) deserted their nest but, most importantly,

259 this abandonment probability was independent of the hormonal manipulation $\left(\chi^{2}=0.23\right.$;

$260 \mathrm{df}=1 ; p=0.63)$ and clutch size $\left(\chi^{2}=0.07 ; \mathrm{df}=1 ; p=0.80\right)$. Decreasing prolactin

261 levels is associated with nest abandonment and lower breeding success in some bird

262 species (Angelier et al., 2016), although this link is not clear in other avian species

263 (Angelier et al., 2016; Kosztolányi et al., 2012; Wojczulanis-Jakubas et al., 2013). Our

264 results confirm previous studies showing that nest desertion is not predicted by changes

265 in circulating prolactin in Eurasian blackbirds (Ruiz-Raya et al., 2018), a potential host

266 that does not use nest desertion as an anti-parasitic defence (Soler et al., 2015). These

267 results suggest that nest abandonment is likely under the control of different

268 neuroendocrine pathways, as well as other important aspects of avian breeding biology 
269 such as energy storage (Angelier et al., 2016; Kosztolanyi et al., 2012). Future studies

270 should explore whether prolactin changes are linked to nest desertion decisions in

271 small-sized hosts that use nest abandonment as anti-parasitic defence. Our experimental

272 manipulation had no impact on females' body mass (sample day: $\mathrm{F}_{1,7}=1.98 ; p=0.20$ )

273 independently on the hormonal treatment (implant treatment $\mathrm{x}$ sample day: $\mathrm{F}_{1,7}=1.02 ; p$

$274=0.35)$. These results indicate that our BRC treatment did not effected females' feeding

275 behaviour (Buntin, 1989), as well as supports previous results showing absence of

276 unintended side effects on feeding behaviour in BRC-treated zebra finches (Taeniopygia

277 guttata) (Smiley and Adkins-Regan, 2018).

278 In conclusion, our findings show that prolactin may play an important role as a mediator

279 of host resistance to avian brood parasitism. While decreasing prolactin levels likely

280 lead to more restrictive acceptance thresholds by facilitating host rejection decisions, the

281 mechanism through which hormonal variations mediate egg-rejection decisions need

282 further exploration. The fact that BRC-treated individuals ejected model eggs at higher

283 rates, as well as the absence of ejection costs, suggests that a reduction in circulating

284 prolactin does not impact the cognitive abilities of hosts. Indeed, changes in the

285 individual cognitive performance are likely mediated by alternative endocrine pathways

286 such as glucocorticoids (Maille and Schradin, 2016). Future studies addressing

287 individual variations in baseline prolactin levels, the strength of the prolactin response

288 to stressors, or differences in target tissue sensitivity to hormones (e.g. prolactin

289 receptor density) would be particularly helpful to further explore the hormonal

290 mechanisms mediating differences in egg-rejection behaviour within and among host

291 populations. Finally, prolactin might also play a decisive role in the regulation of other

292 anti-parasitic host defences linked to parental decisions, such as the rejection of

293 parasitic nestlings (Grim, 2017), so future research on the role of prolactin as mediator 
294 of host defences to brood parasitism should be extended to other stages of the breeding 295 cycle.

296 Ethics. This study follows all relevant Spanish national (Decreto 105/2011, 19 dee 297 Abril) and regional guidelines. No individual exhibited long-term negative effects as a 298 consequence of our treatment.

299 Competing interests. We declare we have no conflict of interest.

300 Authors' contribution. FRR, JDIA and MS designed the study. FRR conducted the 301 fieldwork. CP and OC performed the hormonal assays. FRR analysed the data and 302 wrote the first draft. All authors critically contributed to drafts and gave final approval 303 for publication.

304 Funding. Financial support was provided by Consejería de Economía, Innovación, 305 Ciencia y Empleo; Junta de Andalucía (research project CVI-6653 to MS).

306 References

307 Abolins-Abols, M. and Hauber, M. E. (2018). Host defences against avian brood 308 parasitism: an endocrine perspective. Proc. R. Soc. B Biol. Sci. 285, 20180980.

309 Abolins-Abols, M. and Hauber, M. E. (2020). Endocrine regulation of egg rejection in 310 an avian brood parasite host. Biol. Lett. 16, 20200225.

311 Angelier, F. and Chastel, O. (2009). Stress, prolactin and parental investment in birds: 312 A review. Gen. Comp. Endocrinol. 163, 142-148.

313 Angelier, F., Barbraud, C., Lormée, H., Prud'homme, F. and Chastel, O. (2006).

314 Kidnapping of chicks in emperor penguins: A hormonal by-product? J. Exp. Biol. $315 \quad 209,1413-1420$. 
316 Angelier, F., Wingfield, J. C., Tartu, S. and Chastel, O. (2016). Does prolactin

317 mediate parental and life-history decisions in response to environmental conditions 318 in birds? A review. Horm. Behav. 77, 18-29.

\section{Antonson, N. D., Hauber, M. E., Mommer, B. C., Hoover, J. P. and Schelsky, W.} M. (2020). Physiological responses of host parents to rearing an avian brood parasite: An experimental study. Horm. Behav. 125, 104812.

Bartol, I., Karcza, Z., Moskát, C., Røskaft, E. and Kisbenedek, T. (2002). Responses of great reed warblers Acrocephalus arundinaceus to experimental brood parasitism: the effects of a cuckoo Cuculus canorus dummy and egg mimicry. J. Avian Biol. 33, 420-425.

Buntin, J. D. (1989). Time course and response specificity of prolactin-induced hyperphagia in ring doves. Physiol. Behav. 45, 903-909.

Buntin, J. D. (1996). Neural and hormonal control of parental behavior in birds. $A d v$. Study Behav. 25, 161-213.

\section{Cottin, M., Chastel, O., Kato, A., Debin, M., Takahashi, A., Ropert-Coudert, Y.} and Raclot, T. (2014). Decreasing prolactin levels leads to a lower diving effort but does not affect breeding success in Adélie penguins. Horm. Behav. 65, 134141.

Davies, N. B. and Brooke, M. D. L. (1989). An experimental study of co-evolution between the cuckoo, Cuculus canorus, and its hosts. I. Host egg discrimination. $J$. Anim. Ecol. 58, 207-224.

Feeney, W. E., Welbergen, J. A. and Langmore, N. E. (2014). Advances in the study of coevolution between avian brood parasites and their hosts. Annu. Rev. Ecol. 
Evol. Syst. 45, 227-246.

340

341

342

Goutte, A., Angelier, F., Welcker, J., Moe, B., Clément-Chastel, C., Gabrielsen, G. W., Bech, C. and Chastel, O. (2010). Long-term survival effect of corticosterone manipulation in Black-legged kittiwakes. Gen. Comp. Endocrinol. 167, 246-251.

Grim, T. (2017). Host defences against brood parasite nestlings: theoretical expectations and empirical evidence. In Avian Brood Parasitism Behaviour, Ecology, Evolution and Coevolution (ed. Soler, M.), pp. 539-556. Springer.

Grim, T., Samaš, P., Moskát, C., Kleven, O., Honza, M., Moksnes, A., Røskaft, E. and Stokke, B. G. (2011). Constraints on host choice: why do parasitic birds rarely exploit some common potential hosts? J. Anim. Ecol. 80, 508-518.

Hauber, M. E., Moskát, C. and Bán, M. (2006). Experimental shift in hosts' acceptance threshold of inaccurate-mimic brood parasite eggs. Biol. Lett. 2, 177180.

Kosztolanyi, A., Küpper, C., Chastel, O., Parenteau, C., Yilmaz, K. T., Miklósi, A., Székely, T. and Lendvai, A. Z. (2012). Prolactin stress response does not predict brood desertion in a polyandrous shorebird. Horm. Behav. 61, 734-740.

Kosztolányi, A., Küpper, C., Chastel, O., Parenteau, C., Yilmaz, K. T., Miklósi, Á., Székely, T. and Lendvai, Á. Z. (2012). Prolactin stress response does not predict brood desertion in a polyandrous shorebird. Horm. Behav. 61, 734-740.

Maille, A. and Schradin, C. (2016). Ecophysiology of cognition: How do environmentally induced changes in physiology affect cognitive performance? Biol. Rev. 1102, 1101-1112.

Marchetti, K. (1992). Costs to host defence and the persistence of parasitic cuckoos. 
Proc. R. Soc. - Biol. Sci. 248, 41-45.

363

364

365

Mark, M. M. and Rubenstein, D. R. (2013). Physiological costs and carry-over effects of avian interspecific brood parasitism influence reproductive tradeoffs. Horm. Behav. 63, 717-722.

Moksnes, A., Røskaft, E. and Korsnes, L. (1993). Rejection of cuckoo (Cuculus canorus) eggs by meadow pipits (Anthus pratensis). Behav. Ecol. 4, 120-127.

Moksnes, A., Røskaft, E., Hagen, L. G., Honza, M., Mork, C. and Olsen, P. H. (2000). Common cuckoo Cuculus canorus and host behaviour at reed warbler Acrocephalus scirpaceus nests. Ibis (Lond. 1859). 142, 247-258.

Müller, C., Almasi, B., Roulin, A., Breuner, C. W., Jenni-Eiermann, S. and Jenni, L. (2009). Effects of corticosterone pellets on baseline and stress-induced corticosterone and corticosteroid-binding-globulin. Gen. Comp. Endocrinol. 160, 59-66.

Ouyang, J. Q., Muturi, M., Quetting, M. and Hau, M. (2013). Small increases in corticosterone before the breeding season increase parental investment but not fitness in a wild passerine bird. Horm. Behav. 63, 776-781.

Pinheiro, J., Bates, D., Debroy, S. and Sarkar, D. (2014). nmle: Linear and nonlinear mixed effects models. R package version 3.1-117. Available: http://CRAN.R project.org/package $=$ nlme $>$.

Roldán, M. and Soler, M. (2011). Parental-care parasitism: how do unrelated offspring attain acceptance by foster parents? Behav. Ecol. 22, 679-691.

\section{Roncalli, G., Soler, M., Ruiz-Raya, F., Serrano-Martín, A. J. and Ibáñez-Álamo, J.}

D. (2019). Predation risk affects egg-ejection but not recognition in blackbirds. 
Behav. Ecol. Sociobiol. 73,.

386 Ruiz-Raya, F. and Soler, M. (2017). Phenotypic plasticity in egg rejection: evidence

Smiley, K. O. (2019). Prolactin and avian parental care: New insights and unanswered questions. Horm. Behav. 111, 114-130.

Ruiz-Raya, F., Soler, M., Sánchez-Pérez, L. L. and Ibáñez-Álamo, J. D. (2015). Could a factor that does not affect egg recognition influence the decision of rejection? PLoS One 10, 1-10.

\section{Ruiz-Raya, F., Soler, M., Roncalli, G., Abaurrea, T. and Ibáñez-Álamo, J. D.} (2016). Egg rejection in blackbirds Turdus merula: a by-product of conspecific parasitism or successful resistance against interspecific brood parasites? Front. Zool. 13, 16.

Ruiz-Raya, F., Soler, M., Abaurrea, T., Chastel, O., Roncalli, G. and IbáñezÁlamo, J. D. (2018). Hormonal responses to non-mimetic eggs: is brood parasitism a physiological stressor during incubation? Behav. Ecol. Sociobiol. 72,.

Samas, P., Hauber, M. E., Cassey, P. and Grim, T. (2011). Repeatability of foreign egg rejection: Testing the assumptions of co-evolutionary theory. Ethology 117, $606-619$.

Smiley, K. O. and Adkins-Regan, E. (2018). Lowering prolactin reduces post-hatch 
parental care in male and female zebra finches (Taeniopygia guttata). Horm.

Behav. 98, 103-114.

Sockman, K. W., Sharp, P. J. and Schwabl, H. (2006). Orchestration of avian reproductive effort: an integration of the ultimate and proximate bases for flexibility in clutch size, incubation behaviour, and yolk androgen deposition. Biol. (2013). Decreased prolactin levels reduce parental commitment, egg temperatures, and breeding success of incubating male Adélie penguins. Horm. Behav. 64, 737- 
432 Thorogood, R. and Davies, N. B. (2016). Combining personal with social information 433 facilitates host defences and explains why cuckoos should be secretive. Sci. Rep. 6 , $434 \quad 10-15$.

435 Whittingham, M. J., Stephens, P. A., Bradbury, R. B. and Freckleton, R. P. (2006).

436 Why do we still use stepwise modelling in ecology and behaviour? J. Anim. Ecol.

$437 \quad \mathbf{7 5}, 1182-1189$.

438 Wojczulanis-Jakubas, K., Jakubas, D. and Chastel, O. (2013). Behavioural and 439 hormonal stress responses during chick rearing do not predict brood desertion by $440 \quad$ female in a small Arctic seabird. Horm. Behav. 64, 448-453. 


\section{$452 \quad$ Figure Legends}

453

454

455

(a)

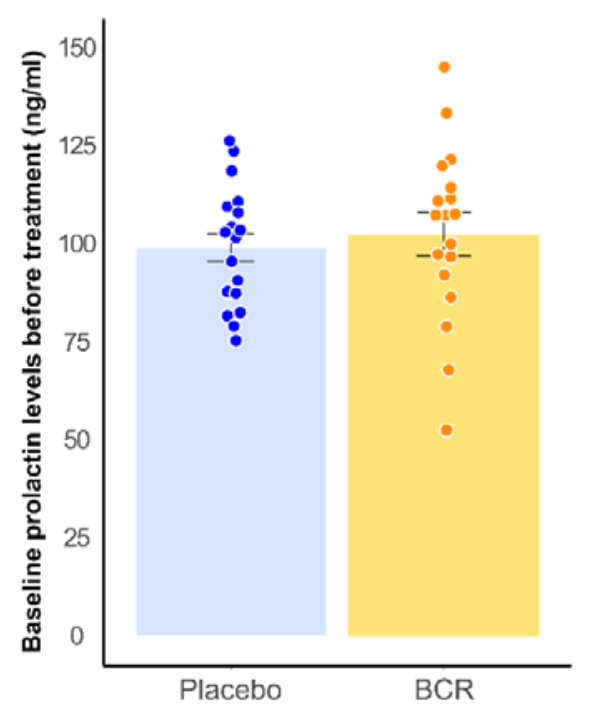

(b)

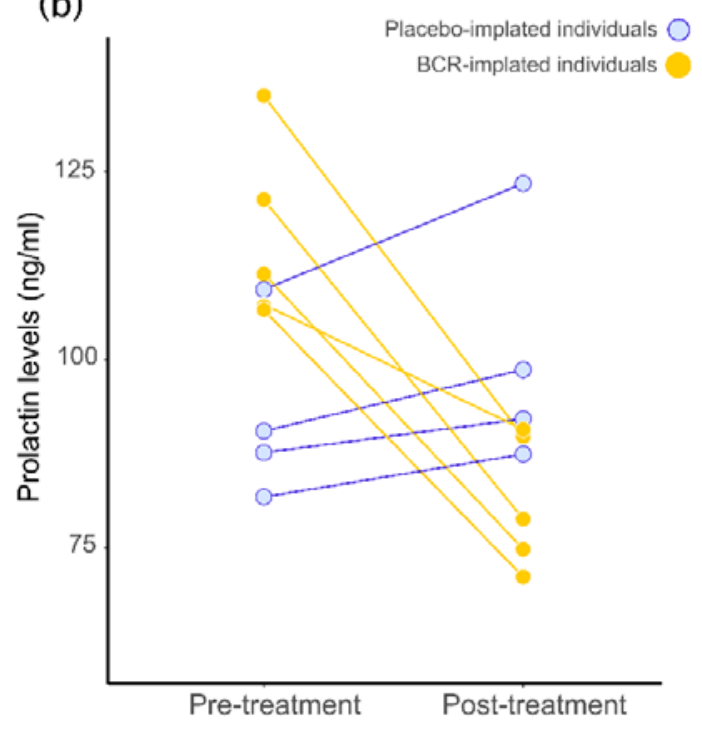

458 Figure 1: (a) Pre-treatment prolactin levels (i.e. before implantation) showed by 459 placebo $(n=18)$ and bromocriptine-implanted (BRC; $n=18)$ females. (b) Effect of the 460 bromocriptine (BRC) 10-days-release pellets on females' prolactin levels 6-days after 461 implantation: BRC-implanted females showed a significant reduction in circulating 462 prolactin post-treatment compared to placebo-implanted individuals. 
(a)

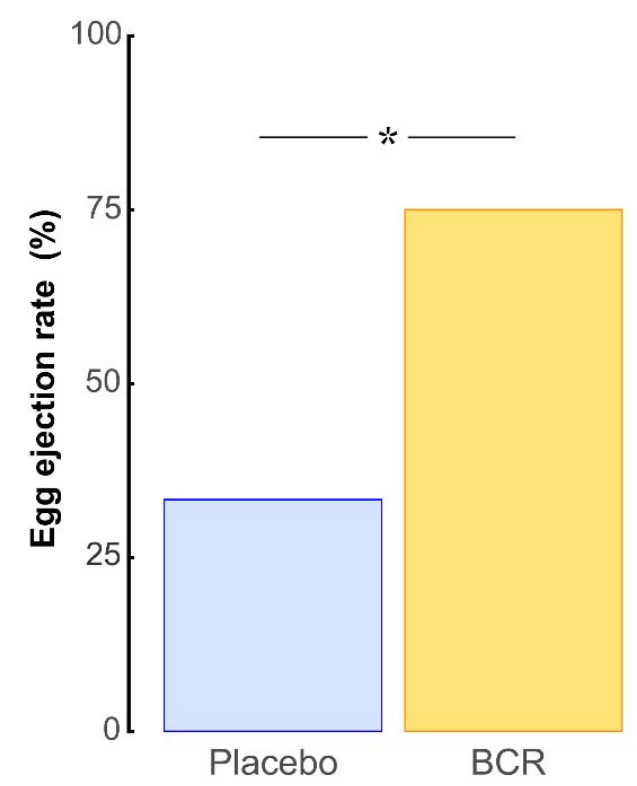

(b)

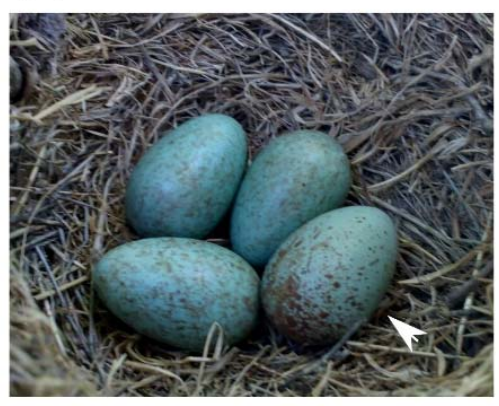

469 Figure 2: (a) Probability of egg ejection in both bromocriptine (BRC; yellow; $\mathrm{N}=16$ )

470 and placebo-implanted females (Placebo; blue; $\mathrm{N}=15$ ). (b) Blackbird clutch 471 experimentally parasitized with a mimetic model egg (white arrow). 